Meta

Journal des traducteurs

Translators' Journal

\title{
La terminologie catalane : bilan des activités
}

\section{Teresa Cabré}

Volume 34, numéro 3, septembre 1989

1. Actes du Colloque Les terminologies spécialisées : Approches quantitative et logico-sémantique et 2 . Actes du Colloque Terminologie et Industries de la langue

URI : https://id.erudit.org/iderudit/002183ar

DOI : https://doi.org/10.7202/002183ar

Aller au sommaire du numéro

Éditeur(s)

Les Presses de l'Université de Montréal

ISSN

0026-0452 (imprimé)

1492-1421 (numérique)

Découvrir la revue

Citer cet article

Cabré, M. T. (1989). La terminologie catalane : bilan des activités. Meta, 34(3),

544-551. https://doi.org/10.7202/002183ar d'utilisation que vous pouvez consulter en ligne.

https://apropos.erudit.org/fr/usagers/politique-dutilisation/ 


\section{LA TERMINOLOGIE CCATALANE: BILAN DES ACTIVITÉS}

TERESA CABré

Université de Barcelone, Barcelone, Espagne

Mon objectif est de présenter un bilan des activités terminologiques en Catalogne, rendant compte de ce qui se fait non seulement dans le cadre de l'administration, mais aussi dans d'autres organismes.

Pour arriver à ce but, je vais faire un inventaire rapide des éléments historiques qui permettent d'expliquer le point de départ des travaux actuels, une brève présentation des activités du moment présent et pour terminer quelques considérations sur la situation actuelle et les perspectives de futur.

On peut dire, en général, qu'en Catalogne, le développement de la terminologie est attaché aux événements historico-politiques et à la situation socio-linguistique. Sans tous ces éléments, on ne peut pas expliquer la situation actuelle.

\section{ANTÉCÉDENTS DES TRAVAUX ACTUELS}

Il faut remonter au début du $\mathrm{XX}^{\mathrm{e}}$ siècle pour rencontrer les fondements et les causes des problèmes terminologiques en Catalogne.

À mon avis, les éléments les plus pertinents qu'il faut retenir sont les suivants :

1.1 Après la crise politique du XVIIIe siècle, la Catalogne commence à se réveiller tant du point de vue économique que culturel. Le XIXe siècle représente l'assimilation en Catalogne des mouvements européens du moment, la renaissance d'une bourgeoisie économiquement active et l'apparition de mouvements culturels et littéraires pareils à ceux qui se produisent en Europe.

1.2 Le Romantisme a réveillé en Europe les sentiments nationalistes des peuples. La Catalogne ne reste pas à l'écart de ces changements rénovateurs et développe un mouvement littéraire en ce sens, la «Renaixença», qui encourage l'usage de la langue catalane, et un mouvement politique nationaliste autour d'un parti politique.

On ne peut pas oublier qu'en 1714, la Catalogne a été envahie par l'armée espagnole et qu'elle a perdu ses institutions politiques et ses lois.

Néanmoins, la population catalane n'a pas renoncé, même pendant cette époque de répression à utiliser la langue catalane comme code habituel de communication familiale.

1.3 La puissante situation économique de la Catalogne, fondée surtout sur les activités commerciales et, au XIX $\mathrm{X}^{\mathrm{e}}$ siècle, sur l'industrialisation, permet de mettre sur pied des projets de développement de la science et de la technique à travers des initiatives aussi bien institutionnelles que civiles.

Trois variantes déterminent donc le réveil terminologique catalan: société civile active et d'un haut niveau culturel, nationalisme et industrialisation.

Ces éléments que je viens d'évoquer, et plusieurs autres que je ne peux pas analyser, expliquent qu'au début du $\mathrm{XX}^{\mathrm{e}}$ siècle, il y avait un intérêt explicite et actif pour la langue catalane dans tous les aspects. Ainsi, un bon nombre de scientifiques et de techni- 
ciens, ayant besoin d'une terminologie pour s'exprimer en langue catalane, ont commencé à la créer dans les Universités. La création, en 1907, de l'Académie de la langue catalane, «l'Institut d'Estudis Catalans», a permis de coordonner les travaux et celui-ci a servi de point de référence pour la normalisation depuis sa création jusqu'à aujourd'hui.

Une des premières tâches de l'IEC a été de codifier la langue et d'établir un modèle normatif. Pour cela, il a créé une équipe de travail, dirigée par Pompeu Fabra, et chargée d'établir l'orthographe normalisée (1913), la grammaire normative (1918) et d'élaborer le dictionnaire de la langue (1932). Toutes ces activités ont été réalisées avant 1932.

P. Fabra, qui était un ingénieur préoccupé par des technicismes et par la langue scientifique et désireux que la langue catalane soit considérée comme une véritable langue de culture au même titre que les autres langues de l'Europe, a introduit dans le dictionnaire de langue générale une quantité considérable de termes de spécialités, qui nous sont d'une grande utilité aujourd'hui.

Les circonstances que je viens de présenter montrent comment l'activité terminologique catalane n'est pas une activité récente, d'où il ressort que l'organisation des travaux actuels ne peut pas oublier les recherches faites dans le cadre du début du XXe siècle.

Il reste encore à faire une analyse systématique de tous ces matériaux terminologiques et une valorisation des résultats.

Avant 1940, il y avait déjà une quarantaine de travaux terminologiques publiés, la plupart au «Butlleti de Dialectologia Catalana» de l'IEC, évidemment de valeur irrégulière. (Annexe 1)

Du point de vue thématique, les travaux se rapportent presque tous à des métiers artisanaux, à des habitudes traditionnelles de la culture populaire, à la culture agricole, aux dénominations populaires en botanique, zoologie, climat, etc. et quelques-uns aux activités industrielles émergentes.

Le caractère particulier de cette période historique justifie cette obsession. Il fallait surtout récupérer les" mots catalans oubliés, sauver la terminologie des métiers en décadence. D'autre part, il fallait mettre à la disposition des usagers les dénominations des activités nouvelles.

En 1931, la II ${ }^{\mathrm{e}}$ République est proclamée en Espagne. Avec cette nouvelle situation politique, le peuple catalan a récupéré son autogouvernement et donc ses droits linguistiques.

Mais la période de normalisation officielle est courte. La République n'a duré que cinq ans, de 1931 à 1936. Le coup d'État du général Franco a supprimé de nouveau tous les droits des autonomies en Espagne.

La répression, affectant la langue, obligera les activités de la recherche linguistique à entrer dans le domaine de la clandestinité.

\section{DE 1940 À 1980 (LE STATUT D'AUTONOMIE)}

Il faut distinguer dans cette longue période deux phases de caractéristiques différentiées que nous pouvons situer autour des années indiquées:

\section{1 de 1940 à 1970}

\section{2 de 1970 à 1980}

\subsection{LA PREMIÈRE PHASE (1940-1970)}

Cette phase se caractérise par le passage d'une clandestinité obligatoire à une ouverture progressive conquise jour après jour, mais avec une élaboration graduelle de travaux «dans et sur» la langue et une disparition progressive de la peur des gens en ce qui concerne l'usage de la langue. 
Après la guerre civile espagnole, la langue catalane est restée interdite officiellement jusqu'à l'approbation de la Constitution espagnole en 1978. Cette interdiction, effective dans la vie officielle et publique, n'a pas empêché de l'utiliser dans la vie privée et de continuer le travail de normalisation du code dans la clandestinité. L'IEC a repris ses activités de travail en 1942. C'est grâce à cette institution que l'on a pu avoir une ligne de continuité de la codification et de l'enseignement de la langue. Il a organisé une sorte de cours universitaires clandestins qui fonctionnèrent jusqu'aux années 1970.

Logiquement, la situation de répression a motivé une forte régression de la recherche sur la langue catalane, et donc de l'élaboration de travaux terminologiques.

Mais progressivement, la situation se récupère au fur et à mesure que le temps passe et les spécialistes commencent de nouveau à travailler en terminologie :

\begin{tabular}{|c|c|c|}
\hline $1940-1950 \ldots$ & avau & approximativement \\
\hline $1950-1960 \ldots \ldots \ldots \ldots$ & " & \\
\hline $1960-1970 \ldots \ldots \ldots$ & $n$ & \\
\hline $1970-1980 \ldots \ldots \ldots \ldots \ldots \ldots . .60$ & $"$ & $"$ \\
\hline $1980-1987 \ldots \ldots \ldots \ldots \ldots . . . .275$ & $"$ & $"$ \\
\hline
\end{tabular}

Dans la période de 1940 à 1950, seulement deux travaux scientifiques d'intérêt terminologique apparaissent en catalan.

Dans la période 1950 à 1960 , il y en a six, tous édités par l'Institut d'Estudis Catalans qui a repris officieusement son activité.

Entre 1960 et 1970, se réalise une production émergente de terminologic. Une quarantaine de travaux sont publiés. La thématique de recherche terminologique s'élargit. Diverses institutions, et non seulement l'IEC, commencent à promouvoir et à publier la terminologie. Une des principales institutions financières catalanes entre activement dans la normalisation de la terminologie bancaire. C'est la première entreprise qui s'occupe de terminologie, quoique non de manière systématique.

L'événement, à mon avis, le plus important pour la terminologie catalane est la parution en 1969 de la Gran Enciclopèdia Catalana, dirigée par Jordi Carbonell, actuellement membre de la Commission de Terminologie. La planification d'un ouvrage des proportions de la GEC a été un élément clé pour la terminologie, parce qu'elle a prévu de mettre à jour les lexiques de spécialité pour fournir à la société catalane un outil de normalisation effective. La GEC est encore aujoud'hui l'outil le plus efficace de consultation terminologique. La parution de la deuxième édition, revisée et élargie, a permis de mettre à jour une partie de la terminologie scientifique et technique catalane. Presque tous les professeurs universitaires de prestige et les scientifiques et techniciens connus ont collaboré à faire ou à superviser la GEC qui a accordé une attention particulière à la terminologie. L'autorité des spécialistes concernés permet de parler d'une certaine normalisation au sens de standardisation des termes proposés.

\subsection{LA DEUXIĖME PHASE (1970-80)}

Cette tranche se caractérise par une récupération progressive des droits politiques et des institutions. En ce sens, il faut mentionner les faits suivants :

$1977 \ldots$ - les premières élections démocratiques ont lieu

- une commission plurale des partis politiques catalans commence à rédiger le texte du Statut d'Autonomie de Catalogne

- l'autogouvernement provisoire est rétabli 
-le président de la Generalitat, qui était exilé en France, revient en Catalogne

1980 Premières élections catalanes

Pendant cette période se produisent des événements pertinents pour la terminologie.

En 1975, un groupe nombreux de scientifiques et techniciens catalans, tous représentatifs de diverses sociétés, organismes institutionnels et ordres professionnels, constituent la Commission Coordinatrice Lexicographique de Sciences ${ }^{1}$ avec le but d'«harmoniser les propositions de termes nouveaux pour désigner des nouveaux concepts et harmoniser les néologismes techniques et scientifiques diffusés».

C'est grâce à la CCLC que la première publication périodique de terminologie, Full lexicographic, apparaît en Catalogne de 1978 à 1982.

La CCLC commence à publier en 1977 une collection de terminologies scientifiques sur les domaines suivants:

mathématiques

physique

chimie

biologie

géologie

anatomie

biochimie

physique médicale

L'IEC, pour sa part, qui — à la différence par exemple de l'Académie espagnole ou de l'Académie française - n'est pas seulement l'organisme chargé de la normativité de la langue, mais est un organisme officiel de la recherche scientifique, optimise la création de sociétés scientifiques, dont la seule langue de travail est le catalan. Ce fait a apporté une quantité importante de terminologie catalane déja normalisée. Aujourd'hui, encore les sociétés de l'IEC sont le point principal de consultation pour les dénominations polémiques des termes nouveaux.

Parallèlement, l'Université Polytechnique de Catalogne commence à travailler sur la terminologie technique.

Ces faits expliquent que l'on puisse parler d'une certaine organisation de la terminologie catalane depuis une date antérieure à son institutionalisation en 1985.

Il faut souligner, comme un élément caractéristique de cette période (1940-1980), que les auteurs de terminologie sont, contrairement à la période précédente, des spécialistes de domaines et non pas des philologues. Leur connaisance du domaine à côté de la connaissance de la structure et de la normativité de la langue faisait d'eux les personnes les plus adéquates, et les principaux intéressés dans ces travaux. En même temps, leur respect pour l'autorité de l'IEC était une garantie de responsabilité linguistique en un moment clé pour la revitalisation et la modernisation de la langue catalane.

\section{DEPUIS 1980 JUSQU'À AUJOURD'HUI}

Plusieurs événements cruciaux se produisent pendant les premières années de la décade actuelle :

- La proclamation de la Constitution espagnole en 1978 qui consacre l'espagnol comme la seule langue officielle de l'état mais qui établit l'officialité des autres langues espagnoles dans leurs territoires respectifs; 
- la proclamation du Statut d'Autonomie en Catalogne, fait qui permet de ressortir les institutions politiques d'autogouvernement et, en ce qui concerne la langue, d'organiser institutionnellement sa normalisation dans tous les domaines;

- la création, en 1980, de la Direction générale de politique linguistique, responsable de l'exécution de la normalisation du Catalan conçue par le gouvernement de Catalogne;

- la proclamation en 1983 de la Loi de normalisation linguistique, dont les objectifs sont les suivants:

a) protéger et stimuler l'usage du catalan pour tous les citoyens;

b) rendre effectif l'usage du catalan;

c) normaliser l'usage du catalan dans tous les moyens de communication de masses;

d) assurer l'extension de la connaissance du catalan.

La Loi de normalisation prévoit trois domaines d'intervention:

L'Administration et autres organismes publics dépendants de la Generalitat

Article 5

1. Le catalan, en tant que langue spécifique de Catalogne, l'est également de la Généralité et de l'Administration territoriale catalane, de l'Administration locale et des autres organismes publics qui dépendent de la Généralité.

2. Le catalan et le castillan, en tant que langues officielles en Catalogne, devront être employées obligatoirement par l'Administration sous la forme établie par la Loi.

\section{L'enseignement}

\section{Article 14}

1. Le catalan, en tant que langue spécifique de Catalogne, l'est également de l'enseignement à tous les niveaux éducatifs.

2. Les enfants ont le droit de recevoir le premier enseignement dans leur langue habituelle, qu'il s'agisse du catalan ou du castillan. L'Administration doit garantir le droit et mettre en ouvre les moyens nécessaires à cette fin. Les parents ou les tuteurs peuvent l'exercer au nom de leurs enfants, en exigeant son application.

3. La langue catalane et la langue castillane devront être obligatoirement enseignées à tous les niveaux et à tous les degrés de l'enseignement non-universitaire.

Les moyens de communication soumis à la responsabilité du gouvernement :

\section{Article 21}

1. La Généralité doit promouvoir la langue et la culture catalanes dans les moyens de communication propres auxquels il est fait référence à l'article 16.3 du Statut d'Autonomie de Catalogne. La langue normalement employée doit être le catalan.

En ce qui concerne le monde du travail, la Loi ne dit que:

\section{Article 25}

1. Le Conseil Exécutif de la Généralité devra encourager la normalisation de l'usage du catalan dans les activités mercantiles, publicitaires, culturelles, associatives, sportives, et de toute autre sorte.

Tandis que tous ces changements politiques et institutionnels se produisent, plusieurs institutions catalanes continuent à travailler en terminologie directement ou indi- 
rectement. Ainsi, les Sociétés de l'IEC continuent à publier des ouvrages scientifiques en langue catalane et, par conséquent, agissent en normalisatrices de la terminologie des domaines scientifiques. Les Universités commencent, ou contineut, à travailler en normalisation.

En 1984, la Direction Générale de la Politique Linguistique organise plusieurs réunions de travail de spécialistes représentatifs des orgnismes concernés par la terminologie dans le but de discuter la possibilité d'organiser des travaux terminologiques en Catalogne. Après ces séances, un séminaire de travail a convoqué un groupe plus restreint de spécialistes pour dessiner le modèle d'organisation de la terminologie catalane. Parallèlement, la DGPL organise le premier cours de formation en terminologie. Il fallait pouvoir disposer d'une équipe de travail pour commencer les travaux institutionnalisés.

Ces faits se produisent à la fin de 1984. L'année suivante, la Generalitat et l'IEC créent TERMCAT, Centre de Terminologie. L'année suivante la Généralitat et l'IEC créent TERCAT, Centre de Terminologie Catalane, avec les objectifs suivants:

- coordonner les activités terminologiques en langue catalane

- diffuser une méthodologie de travail normalisée

- organiser la normalisation de la terminologie nouvelle

- diffuser la terminologie normalisée

- former des terminologues

- représenter la terminologie catalane

La situation sociolinguistique spécifique de la Catalogne requiert la réunion des deux institutions les plus autorisées pour amorcer l'activité. terminologique officielle. Sans l'Institut, la valeur des termes normalisés serait considérablement moindre pour les usagers.

Fonctionnellement TERCAT s'organise à travers :

- une Commission de Surveillance, qui établit les lignes de travail et analyse les travaux réalisés. Deux membres de la DGPL, deux membres de l'IEC, et le Directeur de TERMCAT intègrent cette Commission ;

- un Conseil Superviseur, responsable d'analyser les termes nouveaux et de proposer des formes normalisées. Le Conseil est aussi chargé d'établir les critères linguistiques de la terminologie catalane et la politique des emprunts ;

- le personnel de TERMCAT, avec un directeur qui propose les plans de travail à la Commission de Surveillance et conduit les travaux du Centre.

L'activité des trois premières années des organies de TERMCAT a produit :

- un centre organisé en quatre sections de travail : la terminologie sectorielle, la terminologie ponctuelle et la diffusion, la documentation et les aspects linguistiques de la terminologie;

- un processus de travail terminographique qui mène à la normalisation de la terminologie néologique;

- une méthode pratique, inspirée surtout de celle de l'OLF;

- un projet, la programmation et la réalisation d'une banque générale de données de terminologie spécialisée, maintenant freinée par des problèmes soit financiers, soit de contenu;

- la recherche sur plusieurs sujets relatifs aux critères sur la terminologie;

- environ de 8000 termes nouveaux normalisés;

- la réalisation de cinq cours de formation en terminologie, le dernier faisant partie des cours de troisième cycle offerts par l'Université de Barcelone. 
La présence de l'IEC dans la Commission de Normalisation, garantie d'une certaine expérience de travail et d'une connaissance en profondeur de la langue, donne aux décisions terminologiques une autorité décisive chez nous.

TERMCAT, qui est l'organisme officiel de la terminologie catalane, n'est pas la seule institution dédiée au travail en terminologie: les Universités, à travers leurs Services linguistiques, y travaillent aussi, ainsi que les moyens de communication et plusieurs Services d'autres institutions qui commencent aussi à le faire.

Linguistique structurée, responsable de la résolution des aspects techniques de la normalisation dans les Universités. Ces services linguistiques s'occupent de la traduction espagnol-catalan, de la supervision et de la terminologie.

Le Service Linguistique que je viens d'organiser dans mon université dispose déjà d'un plan de travail à long, moyen et court terme, dans le cadre du Plan général de Normalisation de l'Université de Barcelone.

Sur le plan pratique, chaque Université a organisé son service et ses matériaux de travail en fonction de ses besoins.

Il y a aussi plusieurs entreprises - peu abondantes encore - qui ont commencé à organiser leurs travaux de normalisation, terminologie incluse, mais pour encourager les entreprises à la normalisation, il faudrait à mon avis, une intervention planifiée de l'Administration.

La formation officielle en terminologie, qui était primitivement une activité de l'Administration, s'est déplacée en fait en faveur de l'Université. Elle fait partie des cours des universités et des écoles universitaires selon la spécialité. La formation de terminologues ne se fait qu'à l'Université de Barcelone; la formation de traducteurs, à l'École de Traduction de l'Université Autonome et la formation de documentalistes, à l'école de Bibliotéconomie et de Documentation.

\section{BILAN DE LA SITUATION}

On peut dire que le processus de changement de situation de la langue catalane est un fait réel, initié de façon planifiée à partir de la récupération des institutions politiques, en 1978. La Loi de Normalisation continue son développement.

On peut dire aussi que la terminologie n'a pas été un domaine oublié par la Politique Linguistique. La création en 1985 du Cenre de Terminologie Catalane est une réalité. Mais cette réalité n'a pas encore trouvé, à mon avis, sa cohérence pratique parmi des plans d'intervention institutionnelle. En bref, les plans sur la terminologie ne s'inscrivent pas de façon systématique dans les plans sur la langue appliqués par l'Administration.

En faisant un bilan critique de la situation, je dirais qu'en Catalogne on a travaillé fortement sur l'acceptation de la langue - aujourd'hui la langue catalane est un fait considéré normal et naturel par les gens qui y habitent, quelque soit leur langue, mais à mon avis, le processus n'a pas amorcé la phase ultérieure; je dirais que nous sommes un peu en situation d'impasse, peut-être dû au fait qu'il manque une planification systématique du processus de changement; une planification qui soit clairement exposée et largement partagée et qui inclue des points aussi fondamentaux que :

1. une analyse des besoins;

2. une explicitation des objectifs que l'on veut atteindre;

3. un calendrier approximatif d'exécution;

4. un inventaire des moyens;

5. une définition du plan d'application et une distribution des tâches;

6. la définition préalable de critères d'évaluation des actions menées à terme. 
Malgré tout, le travail déjà fait, il y a, à mon avis, plusieurs points importants encore à résoudre :

a) On ne sait pas quelle est la place ni quel est le degré d'importance que la terminologie doit prendre dans le processus de changement linguistique. La situation de la langue catalane est spécifique et donc, la terminologie doit aussi jouer un rôle spécifique en accord avec la situation. Le modèle de normalisation qu'elle doit suivre ne peut pas être le même que celui appliqué dans d'autres endroits. En Catalogne, la normalisation doit compter sur la terminologie, mais la terminologie qui est, sans doute, un élément important n'est pas l'affaire prioritaire.

b) Il manque encore un modèle de distribution des activités terminologiques qui puisse franchir les frontières de l'institutionnalisation et incorporer la société civile - si importante au début de la terminologie, comme je l'ai dit. L'Administration doit, à mon avis, devenir le moteur d'embrayage et d'optimisation du processus terminologique et non pas le seul agent de travail et le seul représentant d'une orthodoxie figée et immobile. Il faut réfléchir sans cesse sur la théorie, sur les méthodes de travail, sur l'application même, pour réorganiser redistribuer, renouveler ce qui se fait; bref, pour avancer.

c) Ce modèle global sur la terminologie que je viens d'évoquer devrait distribuer les travaux aux diverses institutions, organismes et entreprises selon la préparation scientifique et l'activité habituelle de chacun.

Il y a, je l'ai dit, plusieurs organismes où il faut focaliser, les divers aspects des activités en terminologie:

- la partie linguistique de la normalisation : l'IEC;

- la formation en terminologie : les Universités;

- la réalisation des travaux : les centres de travail sur la langue comme les services linguistiques des universités, les entreprises et l'Administration;

- la terminologie ponctuelle: TERMCAT et les services linguistiques; l'IEC;

- la normalisation conceptuelle: pour le moment les spécialistes nommés par

- la planification, la coordination et la diffusion des activités : TERMCAT.

Celle-ci est - je le pense - la situation de fait, que l'on devrait prendre en considération pour élaborer une planification des travaux terminologiques chez nous.

L'aménagement linguistique, nos amis québécois le savent mieux que nous, ne peut pas devenir une affaire seulement institutionnelle, mais il doit être partagé par le plus grand nombre possible de personnes, parce que ce sont les citoyens, tout compte fait, les véritables agents du changement.

NOTE

Les institutions représentées étaient: l'Académie des Sciences Médicales de Catalogne et Baléares, l'Ordre des pharmaciens, I'Ordre des médecins de Barcelone, la Fondation Torres Ibern, la Société Catalane de Biologie, la Société Catalane des Sciences Physiques, Chimiques et Mathématiques, les trois Sociétés de l'IEC, l'Institut de Technologie du Bâtiment, et l'Institution de Catalane des études agricoles. 